\title{
Analysis of the Prevalence and Severity of Dysregulated Bone Mineral Homeostasis in Nondialyzed Chronic Kidney Disease Patients
}

\author{
Digishaben D. Patel ${ }^{1}$ Uday Vachhani ${ }^{2}$ Ajay Rajput ${ }^{2}$ Pratik Raghavani ${ }^{2}$ Deepak N. Parchwani ${ }^{3}$ \\ Sagar Dholariya ${ }^{3}$
}

${ }^{1}$ Department of Physiology, All India Institute of Medical Sciences,

Address for correspondence Pratik Raghavani, MD, Department of Rajkot, Gujarat, India

${ }^{2}$ Department of Biochemistry, GMERS Medical College, Biochemistry, GMERS Medical College, Himmatnagar, 383001, Himmatnagar, Gujarat, India

Gujarat, India (e-mail: raghavanipratik@gmail.com).

${ }^{3}$ Department of Biochemistry, All India Institute of Medical Sciences, Rajkot, Gujarat, India

J Lab Physicians 2022;14:144-150.

\begin{abstract}
Keywords

- chronic renal insufficiency

- calcium

- phosphorus

- PTH

Background Progressive loss of kidney function in chronic kidney disease (CKD) leads to altered mineral homeostasis, reflected by the imbalance in calcium and phosphorus, and has been associated with progression of renal failure.

Aims The aim of this study was to investigate CKD-mineral bone disorder (CKD-MBD)associated candidate variables and its relationship with parathyroid hormone (PTH), as well as to quantify the prevalence of CKD-associated mineral disturbances in nondialyzed CKD patients.

Materials and Methods This cross-sectional analytical study included 124 CKD patients and 157 control participants. Blood samples were analyzed for serum total calcium, phosphorus, PTH, electrolytes, and other hematological/hemodynamic parameters by standard methods. Suitable descriptive statistics was used for different variables.

Results The 124 patients had a mean age of $50.2 \pm 7.8$ years with male to female ratio of 1.58; majority of patients had stage 3 CKD (40.32\%), and the most common comorbid conditions were diabetes mellitus $(n=78$ [62.9\%]) and hypertension $(n=63$ [50.8\%]). A high prevalence of mineral metabolite abnormalities was observed in a patient cohort; overall prevalence of hyperparathyroidism was found in 57.25\% patients, hypocalcemia in $61.29 \%$, and hyperphosphatemia in $82.25 \%$ patients. Prevalence of abnormal homeostasis (with regard to total calcium, phosphate, and PTH) increased progressively with the severity of disease (analysis of variance; $p<0.05$ ). Significant differences in the mean values of total calcium, phosphorus, alkaline phosphatase, and PTH were seen compared with healthy participants $(p<0.0001)$. Furthermore, there was a significant positive correlation between serum PTH with serum phosphorous $\left(R^{2}: 0.33 ; p<0.0001\right)$, serum creatinine $\left(R^{2}: 0.084 ; p<0.0259\right)$, serum potassium $\left(R^{2}: 0.068 ; p<0.0467\right)$, and a significant negative correlation with serum total calcium $\left(R^{2}: 0.37 ; p<0.0001\right)$.
\end{abstract}

published online July 9, 2021
DOI https://doi.org/ 10.1055/s-0041-1732495. ISSN 0974-2727.

\footnotetext{
(c) 2021. The Indian Association of Laboratory Physicians. All rights reserved.

This is an open access article published by Thieme under the terms of the Creative Commons Attribution-NonDerivative-NonCommercial-License, permitting copying and reproduction so long as the original work is given appropriate credit. Contents may not be used for commercial purposes, or adapted, remixed, transformed or built upon. (https://creativecommons.org/ licenses/by-nc-nd/4.0/)

Thieme Medical and Scientific Publishers Pvt. Ltd., A-12, 2nd Floor, Sector 2, Noida-201301 UP, India
} 
Conclusions CKD patients are at risk of or may already have developed secondary hyperparathyroidism apparent from PTH-linked derangements in mineral metabolism in predialysis CKD patients. These abnormalities start in early stages of CKD and worsen with disease progression. This accentuates the significance of early recognition of mineral bone disorder, understanding its pathophysiological consequences and scheduling necessary interventions/management strategies to protect the CKD patients from a plethora of complications.

\section{Introduction}

Chronic kidney disease (CKD) is characterized by slow progressive abrasion of nephron number or endogenous renal function by means of multifaceted etiologies and is associated with poor outcomes. ${ }^{1,2}$ Outcomes of CKD include rapid progression to end-stage renal disease as well as increased rates of cardiovascular events, cognitive/sexual dysfunction, anemia, mineral, and bone disorders. ${ }^{1-4}$

CKD is stratified into five stages as per Kidney Disease Outcomes Quality Initiative Clinical Practice Guidelines for Chronic Kidney Disease (KDIGO-CKD) ${ }^{1}$ staging and each of these stages is associated with significant perturbations in bone mineral homeostasis, ${ }^{3}$ leading to altered serum concentrations of parathyroid hormone (PTH), phosphorus, calcium, and other markers of disordered bone-mineral metabolism, ${ }^{3}$ with eventual progression to clinical syndrome termed as chronic kidney disease-mineral bone disorder (CKD-MBD). ${ }^{3,4}$

Early detection and management of CKD-associated mineral bone disorder are imperative as it is allied with abnormalities in bone remodeling, renal osteodystrophy, and extraskeletal calcification, ${ }^{3}$ resulting in increased morbidity, augmented all-cause and cardiovascular-specific mortality owing to elevated soft tissue, and vascular and cardiac valvular calcification. ${ }^{3,5,6}$

Over the last two decades or so, numerous clinical and experimental studies have shown abnormal mineral homeostasis and higher mortality in hemodialysis patients. ${ }^{5,6}$ Although these dysregulated bone mineral homeostasis develop early in the course of chronic renal insufficiency, that is, calcium-phosphorus homeostatic abnormalities are already there in patients who have CKD, even prior to commencement of dialysis, ${ }^{1,2}$ their progression and spectrum in predialysis CKD patients are less well described, more so specifically in Gujarati population, in part owing to the lack of comprehensive clinical and biochemical analysis. Thus, this study is an attempt to investigate bone-associated candidate variables and its relationship with PTH as well as to quantify the prevalence of CKD associated mineral disturbances in non-dialyzed CKD patients.

\section{Materials and Methods}

This was a cross-sectional study conducted on newly or previously diagnosed patients of CKD, reporting to the nephrology clinic/medicine outpatient department (OPD) or wards, and the emergency unit of the hospital over a period of 24 months. Inclusion criteria were patients who were $\geq 18$ years of age and patients who have not been started on dialysis or hematinics. Exclusion criteria included patients of acute kidney injury; patients with a history of primary parathyroid disease, hematological neoplasm or solid organ malignancy; patients with chronic infections or patients using any prescription-based calcium supplements, vitamin D analogs, phosphate binders, steroids, anticonvulsants, anticoagulants, calcineurin inhibitors, calcimimetics, bisphosphonates, and other drugs that can affect bone mineral density, in the preceding 12 months; patients taking tobacco or alcohol or having a history of tobacco or alcohol intake (to exclude environmental/external factor for the deterioration of kidney function); pregnant and lactating females; and those patients refusing to sign the consent form.

A total of 281 participants (124 patients and 157 healthy controls) were recruited in the present study after explaining the study protocol and obtaining written informed consent form. Consecutive, convenient sampling technique was used for the enrollment of patient in this study, that is, all the patients who visited the nephrology clinic/medicine OPD or wards of hospital in the study period and fulfilled the criteria were included and it was ensured that 1:1 matching was done. So as per convenience, 124 patients and 157 healthy participants were recruited. Patients were categorized according to the Kidney Disease Outcomes Quality Initiative Clinical Practice Guidelines for Chronic Kidney Disease: Evaluation, Classification, and Stratification. ${ }^{1}$ Healthy controls were selected at random from patients' relatives and participants on routine health checkup who did not have CKD or any significant medical illness. The study was approved by the Institutional Ethical Committee. Data regarding demographics (such as gender, age, education, economic status, residency), occupation, and history suggestive of any systemic illness were collected through a self-administered questionnaire. Blood pressure was measured in the seated position after 10 minutes of rest with a standard manual mercury sphygmomanometer and stethoscope by auscultatory method. ${ }^{7}$ Age was defined as the age at the time of interview (though no documentary proof had been entertained). A sample of venous blood was drawn with an aseptic technique and subjected to estimation of serum total calci$\mathrm{um}^{8}$ serum phosphorus, ${ }^{9}$ serum $\mathrm{PTH}^{10}{ }^{10}$ serum alkaline 
phosphatase, ${ }^{11}$ serum creatinine, ${ }^{12}$ serum urea, ${ }^{13}$ plasma glucose, ${ }^{14}$ serum sodium, ${ }^{15}$ serum potassium, ${ }^{15}$ and hemoglobin. All analysis was done on the same day of collection in a single laboratory using commercial kits.

The statistical analyses were performed using Med Cal statistical software and MS Excel. Data were expressed as mean \pm standard deviation (continuous variables), or as percentages of total (categorical variables). Two-group comparisons were made using chi-squared test $\left(\mathrm{x}^{2}\right)$ for categorical variables and Students' $t$-tests or one-way analysis of variance (ANOVA) for continuous variables. For all analyses, the nominal level of statistical significance was $<0.05$.

\section{Results}

Baseline characteristics are presented in -Table 1 for the patient group and control participants. The mean patient age was $50.2 \pm 7.8$ years. There were 76 males and 48 females, giving a male to female ratio of $1.58: 1$. Diabetes mellitus was present in $62.9 \%$ of patients and $50.8 \%$ patients were hypertensive. Percentage of patients with a history of chronic glomerulonephritis, polycystic kidney disease, and obstructive uropathy was $18.5,7.2$, and $5.6 \%$, respectively ( - Table 1 ). Fifty-two patients had both diabetes and hypertension. Although there was a dominance of male individuals as compared with females in participants ( - Table 1 ), male to female ratio $(p<0.05)$ was statistically similar in patient group and control participants; also there was no statistically significant difference $(p=0.1593)$ in mean age between the studied groups, indicating that the two groups were wellmatched for the analysis in question. - Table 1 also describes the patients as per stratified stages of CKD. The majority of patients had stage 3 CKD (40.32\%), distinctive as glomerular filtration rate between 30 and $60 \mathrm{~mL} / \mathrm{min} / 1.73 \mathrm{~m}^{2}$. In view of the fact that the study enrolled only predialysis CKD patients, it might be the reason for minimal patient in stage $4(11.2 \%)$ and none in stage 5 of CKD (as no reported patient in our hospital setup were without the need of dialysis).

The results of CKD-Bone Mineral Disorder associated indices are summarized in $\mathbf{- T a b l e ~} \mathbf{2}$. The mean values of serum phosphorus were $3.3 \pm 0.8$ and $6.4 \pm 1.7 \mathrm{mg} / \mathrm{dL}$ in control and patients, respectively. The corresponding values for total calcium were $9.6 \pm 1.4$ and $6.7 \pm 1.3 \mathrm{mg} / \mathrm{dL}$, respectively. Mean estimated levels of serum PTH in healthy controls and CKD patients were $44.5 \pm 10.2$ and $364.4 \pm$ $190.6 \mathrm{pg} / \mathrm{dL}$, respectively, that is, the increase in serum PTH levels in patients is statistically significant $(p<0.0001)$. As expected, parameters reflecting renal efficiency (serum creatinine and serum urea) were also significantly elevated in patient cohort as compared with healthy individuals. The mean serum creatinine levels, in controls and CRI patients, were $0.81 \pm 0.09$ and $7.8 \pm 2.4 \mathrm{mg} / \mathrm{dL}$, respectively, while serum urea status in respective group were $23.8 \pm 7.2$ and $138.4 \pm 35.8$.

Table 1 Characteristics of cases and controls participants

\begin{tabular}{|c|c|c|c|c|c|}
\hline \multirow[t]{2}{*}{ Parameters } & $\begin{array}{l}\text { Controls } \\
(n=157)\end{array}$ & $\begin{array}{l}\text { Cases } \\
(n=124)\end{array}$ & \multirow[t]{2}{*}{$t$-Value } & \multirow[t]{2}{*}{$p$-Value } & \multirow[t]{2}{*}{$95 \% \mathrm{Cl}$} \\
\hline & Mean \pm SD & Mean \pm SD & & & \\
\hline Age $(y)$ & $48.8 \pm 8.6$ & $50.2 \pm 7.8$ & 1.41 & 0.15 & -0.55 to 3.35 \\
\hline Sex, males; $n(\%)$ & $88(56.05 \%)$ & $76(61.29 \%)$ & $0.582\left(x^{2}\right)$ & 0.44 & -6.85 to 17.08 \\
\hline Systolic blood pressure $(\mathrm{mm} \mathrm{Hg})$ & $124.8 \pm 6.2$ & $138.8 \pm 18.4$ & 8.91 & $<0.0001$ & 10.91 to 17.09 \\
\hline Diastolic blood pressure $(\mathrm{mm} \mathrm{Hg})$ & $80.2 \pm 2.8$ & $86.2 \pm 3.6$ & 15.71 & $<0.0001$ & 5.25 to 6.75 \\
\hline Fasting plasma glucose (mg/dL) & $80.7 \pm 6.6$ & $102.0 \pm 21.4$ & 11.78 & $<0.0001$ & 17.74 to 24.86 \\
\hline Hemoglobin $(\mathrm{g} / \mathrm{dL})$ & $13.8 \pm 2.8$ & $7.9 \pm 2.1$ & -19.52 & $<0.0001$ & -6.49 to -5.31 \\
\hline \multicolumn{6}{|c|}{ Number (\%) of patients in stratified CKD Stages } \\
\hline Stage $1 ; n(\%)$ & & $18(14.5)$ & & & \\
\hline Stage 2; $n(\%)$ & & $42(33.87)$ & & & \\
\hline Stage 3; $n(\%)$ & & $50(40.32)$ & & & \\
\hline Stage $4 ; n(\%)$ & & $14(11.2)$ & & & \\
\hline Stage $5 ; n(\%)$ & & $00(00)$ & & & \\
\hline \multicolumn{6}{|l|}{ Comorbid conditions } \\
\hline Hypertension; $n(\%)$ & & $63(50.8)$ & & & \\
\hline Diabetes mellitus; $n$ (\%) & & 78 (62.9) & & & \\
\hline Polycystic kidney Disease; $n$ (\%) & & $09(7.2)$ & & & \\
\hline Chronic glomerulonephritis; $n$ (\%) & & $23(18.5)$ & & & \\
\hline $\begin{array}{l}\text { Obstructive } \\
\text { uropathy; n (\%) }\end{array}$ & & $07(5.6)$ & & & \\
\hline
\end{tabular}

Abbreviations: CKD, chronic kidney disease; $\mathrm{x}^{2}$, chi-squared test; $\mathrm{Cl}$, confidence interval; SD, standard deviation. 
Table 2 CKD-bone mineral disorder indices of CKD patients and control groups

\begin{tabular}{|c|c|c|c|c|c|}
\hline \multirow[t]{2}{*}{ Parameters } & Controls $(n=157)$ & Cases $(n=124)$ & \multirow[t]{2}{*}{$t$-Value } & \multirow[t]{2}{*}{$p$-Value } & \multirow[t]{2}{*}{$95 \% \mathrm{Cl}$} \\
\hline & Mean \pm SD & Mean \pm SD & & & \\
\hline Creatinine (mg/dL) & $0.81 \pm 0.09$ & $7.8 \pm 2.4$ & 36.4 & $<0.0001$ & 6.61 to 7.36 \\
\hline Urea $(\mathrm{mg} / \mathrm{dL})$ & $23.8 \pm 7.2$ & $138.4 \pm 35.8$ & 39.13 & $<0.0001$ & 108.84 to 120.36 \\
\hline Alkaline phosphatase & $73.6 \pm 27.2$ & $281.8 \pm 117.6$ & 21.47 & $<0.0001$ & 189.12 to 227.28 \\
\hline Serum PTH (pg/mL) & $44.5 \pm 10.2$ & $364.4 \pm 190.6$ & 21.00 & $<0.0001$ & 289.92 to 349.88 \\
\hline Serum phosphorus (mg/dL) & $3.3 \pm 0.8$ & $6.4 \pm 1.7$ & 20.19 & $<0.0001$ & 2.8 to 3.4 \\
\hline Serum total calcium $(\mathrm{mg} / \mathrm{dL})$ & $9.6 \pm 1.4$ & $6.7 \pm 1.3$ & -17.79 & $<0.0001$ & -3.22 to -2.58 \\
\hline Serum sodium (mEq/L) & $140.5 \pm 4.2$ & $138.8 \pm 5.6$ & -2.90 & 0.0039 & -2.85 to -0.55 \\
\hline Serum potassium (mEq/L) & $4.1 \pm 0.6$ & $4.8 \pm 1.1$ & 6.79 & $<0.0001$ & 0.5 to 0.9 \\
\hline
\end{tabular}

Abbreviations: $\mathrm{Cl}$, confidence interval; CKD, chronic kidney disease; PTH, parathyroid hormone; SD, standard deviation.

- Table 3 reveals the prevalence of abnormal mineral homeostasis in patients as per Kidney Disease Outcomes Quality Initiative Clinical Practice Guidelines for Chronic Kidney Disease (KDIGO-CKD) staging. ${ }^{1}$ Of the 124 patients, there were 76 with state of hypocalcemia. One-hundred two of the 124 patients ( $82.25 \%$ ) were hyperphosphatemic, and hyperparathyroidism was reported in 71 patients. Prevalence of abnormal homeostasis (with regard to total calcium, phosphate and $\mathrm{PTH}$ ) increased progressively with severity of disease (ANOVA; $p<0.05$ ). Serum levels of clinicobiochemical variables in stratified stages of disease are presented in -Table 4. Result suggests that severity of abnormality associated with each of these parameters increased with advanced CKD stage. Serum levels of calcium, phosphorus, potassium, alkaline phosphatase, and creatinine were all strongly associated with the PTH (-Table 5). However, no correlation could be established between the PTH and serum sodium or urea levels.

\section{Discussion}

Animal models ${ }^{16,17}$ have convincingly demonstrated boneassociated abnormal mineral homeostasis in uremia; few clinical and experimental studies in humans also have explored this. ${ }^{18-20}$ Even though these disturbances commence in earlier stages of CKD, their progression in predialysis CKD patients, particularly to stratified stages of CKD, has been contrasting. Therefore, in this study, it is considered worthwhile to assess markers of disordered bone and min- eral metabolism as well as to evaluate the prevalence of CKD associated mineral disturbances and found significant derangements of bone mineral homeostasis in the nondialyzed CKD patients. Our findings support the work of other researchers. ${ }^{18-22}$ Among the inducted patient cohort, most commonly stated symptoms (related to CKD-MBD) were bone pains and myalgias, observed in $41.93 \%$ ( $n: 52$ ) of patients and most common comorbid conditions were diabetes mellitus ( $n=78$ [62.9\%]) and hypertension $[n=63$ [50.8\%]).

We observed an overall prevalence of hyperparathyroidism in $57.25 \%$ patients, hypocalcemia in $61.29 \%$, and hyperphosphatemia in $82.25 \%$ of patients. Similar biochemical abnormalities were reported by few studies. For example, in 2016, Vikrant and Parashar ${ }^{23}$ reported hypocalcemia in $23.8 \%$, hyperphosphatemia in $55.4 \%$, and secondary hyperparathyroidism in $82.7 \%$ patients; and Agarwal ${ }^{21}$ illustrated hypocalcemia, hyperphosphatemia, and hyperparathyroidism in 29.9, 45, and $57.8 \%$ of CKD stage 4 patients, respectively. Progressive loss of kidney function in CKD leads to altered mineral homeostasis, that is reflected by an increasing prevalence of mineral disturbance with the advancing stages of CKD (- Table 3). Moreover, prevalence of aforementioned variables disturbances at each stage was significantly higher from its preceding stage ( - Table 3; ANOVA, $p<0.05$ ), substantiated by the observation that hypocalcemia (78.57\%), hyperparathyroidism (85.71\%), and hyperphosphatemia (92.85\%) were more prevalent in CKD stage 4 patients than any of the preceding stages of CKD (- Table 3). Similar

Table 3 Prevalence of total calcium, phosphorus, and PTH disturbance in stratified stages of CKD $(n=124)$

\begin{tabular}{|l|l|l|l|}
\hline Stages of CKD & Hypocalcemia, $\boldsymbol{n}(\%)$ & Hyperphosphatemia, $\boldsymbol{n}(\%)$ & Hyperparathyroidism, $\boldsymbol{n}(\%)$ \\
\hline 1 & $7(38.88 \%)$ & $10(55.55 \%)$ & $6(33.33 \%)$ \\
\hline 2 & $23(54.76 \%)$ & $35(83.33 \%)$ & $21(50.00 \%)$ \\
\hline 3 & $35(70.00 \%)$ & $44(88.00 \%)$ & $32(64.00 \%)$ \\
\hline 4 & $11(78.57 \%)$ & $13(92.85 \%)$ & $12(85.71 \%)$ \\
\hline Total & $76(61.29 \%)$ & $102(82.25 \%)$ & $71(57.25 \%)$ \\
\hline
\end{tabular}

Abbreviations: CKD, chronic kidney disease; PTH, parathyroid hormone. 
Table 4 CKD-bone mineral disorder indices of patients in stratified stages of CKD

\begin{tabular}{|l|l|l|l|l|l|}
\hline \multirow{2}{*}{ Parameters } & Stage 1 & Stage 2 & Stage 4 & Stage 4 & ANOVA \\
\cline { 2 - 6 } & Mean \pm SD & Mean \pm SD & Mean \pm SD & Mean \pm SD & $p$-Value \\
\hline Creatinine $(\mathrm{mg} / \mathrm{dL})$ & $3.3 \pm 1.1$ & $6.8 \pm 3.1$ & $8.6 \pm 2.9$ & $9.1 \pm 1.8$ & $<0.001$ \\
\hline Urea $(\mathrm{mg} / \mathrm{dL})$ & $78.5 \pm 27.1$ & $127.3 \pm 33.6$ & $145.8 \pm 57.2$ & $157.1 \pm 66.5$ & $<0.001$ \\
\hline Alkaline phosphatase & $202 \pm 111.2$ & $243.2 \pm 107.7$ & $298.3 \pm 131.9$ & $327.2 \pm 133.1$ & $<0.01$ \\
\hline Serum PTH $(\mathrm{pg} / \mathrm{mL})$ & $141.4 \pm 47.2$ & $233.3 \pm 128.6$ & $414.8 \pm 187.5$ & $451.1 \pm 203.3$ & $<0.001$ \\
\hline Serum phosphorus (mg/dL) & $3.9 \pm 0.6$ & $4.6 \pm 1.2$ & $6.9 \pm 1.4$ & $7.7 \pm 1.8$ & $<0.001$ \\
\hline Serum total calcium (mg/dL) & $7.4 \pm 1.6$ & $6.9 \pm 1.3$ & $6.3 \pm 1.0$ & $6.0 \pm 0.8$ & $<0.01$ \\
\hline Serum sodium (mEq/L) & $141.3 \pm 4.2$ & $137.1 \pm 5.2$ & $133.5 \pm 6.1$ & $124.6 \pm 4.1$ & $<0.05$ \\
\hline Serum potassium (mEq/L) & $4.3 \pm 0.7$ & $4.6 \pm 1.4$ & $4.9 \pm 1.3$ & $5.0 \pm 0.7$ & $<0.05$ \\
\hline
\end{tabular}

Abbreviations: ANOVA, analysis of variance; CKD, chronic kidney disease; PTH, parathyroid hormone; SD, standard deviation.

findings were reported by earlier researchers ${ }^{22,23}$ that mineral disturbances were higher in patients with CKD stage 5 than CKD stage 4. Among the studied indices, most prevalent abnormality was hyperphosphatemia, accounting for $82.25 \%$ of the patients.

The mean value of serum phosphors in CKD patients was significantly higher than in healthy controls $(p<0.0001$; - Table 2). This finding complements earlier studies ${ }^{20,22,23}$ that demonstrated levels of phosphorus concentration in newly detected advanced renal failure patients to be high. On contrary to serum phosphorus levels, - Table 2 reveals a significant decrease in the average values of serum total calcium in patient group in contrast to controls. Levels of total calcium concentration in CKD patients have been found to be low in studies conducted in varied geographical areas. ${ }^{19-23}$ Furthermore, it was observed that serum PTH has linear negative correlation with serum total calcium $\left(R^{2}: 0.37 ; p<\right.$ 0.0001; - Table 5) and linear positive correlation with serum phosphorous $\left(R^{2}: 0.33 ; p<0.0001\right.$; - Table 5). In the study, it was also found that the serum PTH $(331.68 \pm 204.99 \mathrm{pg} / \mathrm{mL})$ was significantly higher in patient group as compared with controls ( - Table 2; $p<0.0001$ ) and there was a significant progressive increase in levels of PTH with an advancing stage of CKD ( - Table 4; ANOVA, $p<0.0001$ ), which substantiates the relationship between severity of hyperparathyroidism and the degree of renal impairment. These findings are in accor-

Table 5 Correlation coefficients for PTH with various biochemical measurements

\begin{tabular}{|l|l|l|}
\hline Parameters & $R^{2}$ & $p$-Value \\
\hline Serum total calcium & 0.37 & $<0.0001$ \\
\hline Serum phosphorus & 0.33 & $<0.0001$ \\
\hline Serum sodium & 0.074 & 0.1207 \\
\hline Serum potassium & 0.068 & 0.0467 \\
\hline Serum alkaline phosphatase & 0.59 & $<0.001$ \\
\hline Serum urea & 0.061 & 0.3556 \\
\hline Serum creatinine & 0.084 & 0.0259 \\
\hline
\end{tabular}

Abbreviations: PTH, parathyroid hormone; $\mathrm{R}$, correlation coefficient. dance with Levin et $\mathrm{al}^{19}$ in 2007, Vikrant and Parashar ${ }^{23}$ in 2016, and Arora et al (2018). ${ }^{24}$

The pathophysiology behind the observed effect seems to be secondary hyperparathyroidism resultant to CKD. With declining kidney function, the first event that usually occurs is the declined filtration and excretion of phosphate ensuing in hyperphosphatemia. ${ }^{24,25}$ In the beginning, it is overcome by increase in serum PTH levels, which reduces reabsorption of phosphate from proximal tubules. ${ }^{25}$ However, in due course there is hyperplasia and hypertrophy of the parathyroid gland, setting the stage for secondary hyperparathyroidism. $^{25}$ Concurrently, with progressive decline in kidney function, paucity of activated vitamin $D$ occurs due to decrease of renal 1-hydroxylase activity. ${ }^{26}$ Under normal physiological conditions, calcitriol decreases PTH secretion primarily by stimulating the VDR (expressed in the parathyroid glands) and by down regulating the synthesis of PTH as a result of an effect at the level of transcription of the PTH gene. ${ }^{25,26}$ Both of these changes, that is, diminished filtration/excretion of phosphate and scarcity of activated vitamin $\mathrm{D}$, stimulate an increase in PTH synthesis and secretion. In addition, fibroblast growth factor-23 (FGF23), micro-RNAs, Wnt-b-catenin molecular signaling pathway, epidermal growth factor receptor (EGFR), and transforming growth factor- $\alpha$ (TGF- $\alpha$ ) are suggestive conceivable molecular reasons for the connotation of hyperparathyroidism in CKD. FGF-23 elevates in CKD to regulate phosphate level, but increased FGF-23 leads to decreased production of calcitriol in kidney, which in turn causes initiation/progression of secondary hyperparathyroidism. ${ }^{27}$ Wnt binds with receptor complex of frizzled-LRP5/6, which stimulates bone development and differentiation. Inhibitors of Wnt-b-catenin signaling pathway (namely, Dickkopf-1 and Sclerostin) were found to be associated with dysregulated mineral metabolism with subsequent hyperparathyroidism in patients of CKD. ${ }^{28}$ With repute to EGFR and TGF- $\alpha$, increased expression of EGFR and TGF- $\alpha$ in parathyroid gland of patients with CKD is reported, which in turn results in polyclonal hyperplasia and hyperparathyroidism. ${ }^{28,29}$ Upregulation of miR-21, miR-148, miR-29, miR-30, and miR-141 and downregulation of miR-10, miR-25, and miR-125 have 
been associated with hyperparathyroidism in experimental model, providing an indication of their role in CKD induced hyperparathyroidism. ${ }^{29}$

Hypocalcemia (a finding in current study; - Tables 2 and $\mathbf{3}$ ) is a powerful stimulus for PTH secretion and for parathyroid growth. ${ }^{25,30}$ Calcium exerts negative feedback on PTH secretion through the calcium sensing receptors on the parathyroid. ${ }^{25,30}$ Decrease in serum calcium during the course of CKD caused by phosphate retention and decreased 1,25 dihydroxy cholecalciferol attenuate this feedback and lead to increased PTH mRNA levels and proliferation of parathyroid cells. ${ }^{30}$ The number of calcium sensing receptors also may decrease in hypertrophied parathyroid tissue and lead to inadequate suppression of PTH secretion even in the setting of normal or high calcium levels. ${ }^{30}$ This explains for the increase in phosphorus level and corresponding decrease in the calcium levels in present study.

The mean value of serum sodium and serum potassium in this study was $138.8 \pm 5.6 \mathrm{mEq} / \mathrm{L}$ and $4.8 \pm 1.1 \mathrm{mEq} / \mathrm{L}$, respectively, in CKD patients. Also, it was found that these values were significantly different (-Table 2 ) from the observed values in healthy control population. There was no statistical significant difference noticed among healthy controls and CKD patients with reference to diet, physical activity, and any other lifestyle variation. However, patients had higher plasma glucose $(p<0.0001)$, systolic blood pressure readings ( $p<0.0001)$, and diastolic pressure $(p<$ 0.0001 ) as compared with healthy counterparts, in spite of being under medication, probably because diabetes mellitus and hypertension were found to contribute maximally to the patients as a comorbid disease (-Table $\mathbf{1}$ ).

The observed findings of altered levels of calcium, phosphorus, and PTH not only result to bone mineral defects/disease but also may lead to extraskeletal calcification and potentially calciphylaxis, ${ }^{31}$ causing other harmful repercussion. In the serum, as the level of one or both ions elevates, there is an amplified risk for an ionic bond to form, generating an insoluble complex, which actually aggravates the effects of coronary atherosclerosis through increased vascular calcification and smooth muscle proliferation. ${ }^{31,32}$ Elevated phosphorus may alter microcirculatory hemodynamics through increased extravascular resistance and further compromise myocardial perfusion. Vascular calcification, manifested as reduced vessel wall elasticity, increased intima-media layer thickness, or enhanced pulse-wave velocity, individually or collectively leads to left ventricular hypertrophy. ${ }^{31,32}$ Thus, biochemical indices such as PTH, calcium, phosphate, and other associated parameters must be sustained within target ranges to prevent bone disease, extraskeletal calcification, and to maintain homeostasis of other body systems.

To summarize and conclude, our study reveals a PTHlinked derangements in mineral metabolism apparent from hyperphosphatemia and hypocalcemia in predialysis CKD patients. These abnormalities start in early stages of CKD and worsen with disease progression. This accentuates the significance of early recognition of mineral bone disorder, understanding its pathophysiological consequences and scheduling necessary interventions/management strategies to protect the CKD patients from a plethora of complications.

Nonetheless, a few limitations of our study have to be acknowledged. First, it was a hospital-based study in a referral center, with a stringent selection criteria and voluntary recruitment; thus, sampling may not be a true reflection of the patient community. Hence, data need to be cautiously generalized to the entire population of patients with CKD. Second, the study was cross-sectional in nature and hence, we are not able to infer cause effect relationship between PTH levels and CKD, but confirm the need to monitor PTH and mineral levels from the early stages of CKD. Another limitation is that single measures of calcium, phosphate, and PTH were done. Moreover, few studies suggest prospective changes in bone mineral metabolic parameters; therefore, use of a single value has not been validated.

In summary, results of the study have shown CKD patients are at risk of or may already have developed secondary hyperparathyroidism; hence, findings of this study have implications for the care of CKD patients. Patients and care providers should give the highest priority for early identification and treatment of secondary hyperparathyroidism. It is crucial to preventing or controlling the consequences of this complication. If this can be achieved, the number of patients, in whom plethora of complications develops, should decline. However, additional prospective studies and clinical trials are warranted to clarify the role of secondary hyperparathyroidism in progression of CKD.

\section{Conflict of Interest}

None.

\section{References}

1 National Kidney Foundation. K/DOQI clinical practice guidelines for chronic kidney disease: evaluation, classification, and stratification. Am J Kidney Dis 2002;39(2 Suppl 1):S1-S266

2 National Kidney Foundation. KDOQI Clinical Practice Guidelines and Clinical Practice Recommendations for Anemia in Chronic Kidney Disease KDOQI. Am J Kidney Dis 2006;47:S1-S145

3 Kidney Disease: Improving Global Outcomes (KDIGO) CKD-MBD Work Group. KDIGO clinical practice guideline for the diagnosis, evaluation, prevention, and treatment of chronic kidney diseasemineral and bone disorder (CKD-MBD. Kidney Int Suppl 2009; 113:S1-S130

4 Bello AK, Alrukhaimi M, Ashuntantang GEet al.Complications of chronic kidney disease: current state, knowledge gaps, and strategy for action. Kidney Int Suppl (2011 2017;7(02):122-129

5 Tentori F, Blayney MJ, Albert JMet al.Mortality risk for dialysis patients with different levels of serum calcium, phosphorus, and PTH: the Dialysis Outcomes and Practice Patterns Study (DOPPS. Am J Kidney Dis 2008;52(03):519-530

6 Block GA, Klassen PS, Lazarus JM, Ofsthun N, Lowrie EG, Chertow GM. Mineral metabolism, mortality, and morbidity in maintenance hemodialysis. J Am Soc Nephrol 2004;15(08):2208-2218

7 Rose GA, Blackburn H. Cardiovascular survey methods. Monogr Ser World Health Organ 1968;56:1-188

8 Morin LG. Direct colorimetric determination of serum calcium with o-cresolphthalein complexon. Am J Clin Pathol 1974;61(01): 114-117

9 Daly JA, Ertingshausen G. Direct method for determining inorganic phosphate in serum with the "CentrifiChem". Clin Chem 1972;18(03):263-265 
10 Blind E. Measurement of intact parathyroid hormone by an extracting two-site immunometric assay. In: Schmidt-Gayk $\mathrm{H}$, Armbruster FP, Bouillon R, eds. Calcium Regulating Hormones, Vitamin D Metabolites, an Cyclic AMP. Heidelberg: Springer; 1990:151

11 Wenger C, et al. Alkaline phosphatase. In: Kaplan A, et al. Clin Chem The C.V.. St Louis, Toronto: Mosby Co; 1984:1094-1098

12 Chasson AL, Grady HJ, Stanley MA. Determination of creatinine by means of automatic chemical analysis. Tech Bull Regist Med Technol 1960;30:207-212

13 Chaney AL, Marbach EP. Modified reagents for determination of urea and ammonia. Clin Chem 1962;8:130-132

14 Trinder P. Determination of blood glucose using an oxidaseperoxidase system with a non-carcinogenic chromogen. J Clin Pathol 1969;22(02):158-161

15 Kruse-Jarres JD. Ion-selective potentiometry in clinical chemistry. A review. Med Prog Technol 1988;13(03):107-130

16 Davies MR, Lund RJ, Hruska KA. BMP-7 is an efficacious treatment of vascular calcification in a murine model of atherosclerosis and chronic renal failure. J Am Soc Nephrol 2003;14(06):1559-1567

17 Cortadellas O, Fernández del Palacio MJ, Talavera J, Bayón A. Calcium and phosphorus homeostasis in dogs with spontaneous chronic kidney disease at different stages of severity. J Vet Intern Med 2010;24(01):73-79

18 Kestenbaum B, Belozeroff V. Mineral metabolism disturbances in patients with chronic kidney disease. Eur J Clin Invest 2007;37 (08):607-622

19 Levin A, Bakris GL, Molitch Met al.Prevalence of abnormal serum vitamin D, PTH, calcium, and phosphorus in patients with chronic kidney disease: results of the study to evaluate early kidney disease. Kidney Int 2007;71(01):31-38

20 Schwarz S, Trivedi BK, Kalantar-Zadeh K, Kovesdy CP. Association of disorders in mineral metabolism with progression of chronic kidney disease. Clin J Am Soc Nephrol 2006;1(04):825-831

21 Agarwal SK. Assessment of renal bone mineral disorder in naïve CKD patients: A single center prospective study. Indian J Nephrol 2007;17:96
22 Etta PK, Sharma RK, Gupta A. Study of chronic kidney diseasemineral bone disorders in newly detected advanced renal failure patients: a hospital-based cross-sectional study. Saudi J Kidney Dis Transpl 2017;28(04):874-885

23 Vikrant S, Parashar A. Prevalence and severity of disordered mineral metabolism in patients with chronic kidney disease: a study from a tertiary care hospital in India. Indian J Endocrinol Metab 2016;20(04):460-467

24 Arora K, Goyal G, Soin D, Kumar S, Arora H, Garg C. Correlation of parathyroid hormone levels with mineral status in end-stage renal disease patients. Indian J Endocrinol Metab 2018;22(06): 735-739

25 Tomasello S, Pharm D. Secondary hyperparathyroidism and chronic kidney disease. Diabetes Spectr 2008;21:19-25

26 Holick MF. Vitamin D for health and in chronic kidney disease. Semin Dial 2005;18(04):266-275

27 Mazzaferro S, Pasquali M, Tartaglione L, Rotondi S, Pirrò G Fisiopatologia dell'iperparatiroidismo secondario: ruolo di FGF23 e Klotho. [Pathophysiology of secondary hyperparathyroidism: the role of FGF23 and Klotho]G Ital Nefrol 2009;26(Suppl 49):S11-S17

28 Cejka D, Herberth J, Branscum AJet al.Sclerostin and Dickkopf- 1 in renal osteodystrophy. Clin J Am Soc Nephrol 2011;6(04):877-882

29 Shilo V, Mor-Yosef Levi I, Abel Ret al.Let-7 and MicroRNA-148 regulate parathyroid hormone levels in secondary hyperparathyroidism. J Am Soc Nephrol 2017;28(08):2353-2363

30 Naveh-Many T, Rahamimov R, Livni N, Silver J. Parathyroid cell proliferation in normal and chronic renal failure rats. The effects of calcium, phosphate, and vitamin D. J Clin Invest 1995;96(04): 1786-1793

31 London GM, Guérin AP, Marchais SJ, Métivier F, Pannier B, Adda H. Arterial media calcification in end-stage renal disease: impact on all-cause and cardiovascular mortality. Nephrol Dial Transplant 2003;18(09):1731-1740

32 Raggi P, Boulay A, Chasan-Taber Set al.Cardiac calcification in adult hemodialysis patients. A link between end-stage renal disease and cardiovascular disease? J Am Coll Cardiol 2002; 39(04):695-701 